\title{
Effects of application of exogenous NO on the physiological characteristics of perennial ryegrass grown in $\mathrm{Cd}$ - contaminated soil
}

\author{
W.W. Wang, X.Y. Bai, Y.J. Dong*, W.F. Chen, Y.L. Song, X.Y. Tian \\ College of Resources and Environment, Shandong Agricultural University, Tai'an 271018, P.R. China \\ *Corresponding author: yjdong@sdau.edu.cn
}

\begin{abstract}
A pot experiment was conducted to study the effects of sodium nitroprusside (SNP, a nitric oxide [NO] donor) on cadmium $(\mathrm{Cd})$ toxicity in ryegrass plants. In the present study, ryegrass plants subjected to Cd toxicity were treated with different concentrations of SNP $(50,100,300$ and $500 \mu \mathrm{M})$, SNP solution was poured into Cdcontaminated soil every seven days. Cd treatment inhibited the plants growth and the absorption of micronutrients, increased accumulation of $\mathrm{Cd}$ in both shoots and roots. In Cd-treated plants, the activities of antioxidant enzymes and the contents of proline, soluble protein and ascorbic acid (AsA) decreased dramatically, whereas the accumulation of superoxide anion $\left(\mathrm{O}_{2}{ }^{-}\right)$, hydrogen peroxide $\left(\mathrm{H}_{2} \mathrm{O}_{2}\right)$ and malondialdehyde (MDA) increased significantly. Addition of SNP significantly decreased $\mathrm{O}_{2} \cdot \mathrm{H}_{2} \mathrm{O}_{2}$ and MDA accumulation in both shoots and roots of Cd-stressed plants, decreased the transport of $\mathrm{Cd}$ from roots to shoots, alleviated the inhibition of $\mathrm{Ca}$, $\mathrm{Fe}, \mathrm{Mg}$ and $\mathrm{Zn}$ absorption induced by $\mathrm{Cd}$ and promoted the plant growth. In SNP-treated plants, the chlorophyll content, the contents of proline, soluble protein and ascorbic acid, and the activities of superoxide dismutase, peroxidase and catalase increased considerably. Addition of $300 \mu \mathrm{M}$ SNP had the most significant alleviating effect against $\mathrm{Cd}$ toxicity while the addition of $500 \mu \mathrm{M}$ SNP had no significant effect with $\mathrm{Cd}$ treatment. Based on these results, we concluded that the optimal concentrations of exogenous SNP could alleviate Cd induced stress and promote ryegrass plant growth.
\end{abstract}

Keywords: Cadmium, nitric oxide, ryegrass, antioxidative systems, ion accumulation

Abbreviations: SNP, sodium nitroprusside, NO, nitric oxide, Cd, cadmium, SOD, superoxide dismutase, POD, peroxidase, CAT, catalase, ROS, reactive oxygen species, $\mathrm{O}_{2}^{-}$, superoxide anion radical, $\mathrm{H}_{2} \mathrm{O}_{2}$, hydrogen peroxide; MDA, malondialdehyde, AsA, ascorbic acid 


\section{Introduction}

In recent years, soil pollution was becoming more and more severe due to the increased using in mining, manufacturing and of synthetic products, such as pesticides, paints, batteries, industrial waste and land application of industrial or domestic sludge (Fang and Wang, 2000). And contamination of the environment with toxic heavy metals, such as cadmium $(\mathrm{Cd})$, is an important environmental and human health problem. $\mathrm{Cd}$ is rather mobile in soil and contaminates food and fodder crops even at low soil levels. Since Cd is also not naturally biodegradable, it is persistent once released into the environment and hence $\mathrm{Cd}$ removal from both soil and water is an important environmental protection issue (Chen et al., 2013). Cadmium can interfere with enzymatic activities, membrane functions, and hormone balance. One of the main toxic effects of $\mathrm{Cd}$ is oxidative stress. Oxidative stress is defined as all of the effects such as cellular damage, caused by the active forms of oxygen such as superoxide $\left(\mathrm{O}_{2}^{-{ }^{-}}\right)$, hydrogen peroxide $\left(\mathrm{H}_{2} \mathrm{O}_{2}\right)$, hydroxyl radical $\left(\mathrm{OH}^{-}\right)$and singlet oxygen $\left({ }^{1} \mathrm{O}_{2}\right)$. As the results of oxidative stress, lipid is peroxided, protein synthesis is inhibited, enzyme is inactivated, and membrane systems are damaged.

Nitric oxide (NO) exists in plant cells and intercellular spaces widely and it is a ubiquitous signal molecule involved in multiple plant responses to environmental stress (He et al., 2012). Under stress conditions, such as salinity (Dong et al., 2015), drought (Durán et al., 2016), heavy metal toxicity (Rady and Hemida, 2015), plants produce active oxygen species, which are harmful to plant growth and productivity. Different studies have particularly demonstrated NO signalling in the induction of cell death, defense genes and interaction with reactive oxygen species (ROS) during plant defense against pathogen attack (Wendehenne et al., 2001; Neill et al., 2003). These studies suggest that NO might have acted as an antioxidant for scavenging reactive oxygen species (ROS) generated under the abiotic stress conditions. Plants have evolved a complex antioxidant system (i.e., enzymatic and nonenzymatic detoxification mechanisms) for protecting potential cell injury against tissue dysfunction (Srivastava et al., 2004). The antioxidant enzymes, such as catalase (CAT), peroxidases (POD), superoxide dismutases (SOD), etc., can scavenge different types of ROS. NO also might be involved in increasing the antioxidant content and antioxidative enzymes activity in plants. In recent decade, the effects of exogenous $\mathrm{NO}$ on alleviating heavy metal toxicity in plants were reported by many authors (Khairy et al., 2016; Saxena and Shekhawat, 2013). Much of the published research concerned with NO has important roles in modulating the plant responses to $\mathrm{Cd}$ toxicity (Liu et al., 2015; Kováčik et al., 2015). However, little knowledge is reported regarding the effects of application of exogenous $\mathrm{NO}$ in Cd-contaminated soil on plant growth and development in the whole growth period, and further, or how the growing plants cope with $\mathrm{Cd}$ toxicity through physiological and biochemical adaptations.

Perennial ryegrass is one of the most widely used perennial grasses in temperate regions around the world. Perennial ryegrass can accumulate metals in its biomass, and commonly used as a suitable species for revegetation of metalliferous wastes (Arienzo et al., 2004). Previous researches demonstrated that perennial ryegrass has potential for rehabilitation of Cd-contaminated soils (Arienzo et al., 2004; Lou et al., 2013). In addition, better understanding of Cd-induced oxidative stress and Cd-accumulation in perennial ryegrass can provide important information for phytoremediation. Our previous studies have demonstrated that NO may ameliorate toxic effects of excess 
Cd on lettuce seedlings (Xu et al., 2014) and on ryegrass seedlings grown in Cd-treated nutrient solutions (Wang et al., 2013). Based on the above studies, the objective of this study was to determine the application of exogenous NO could alleviate Cd toxicity, increase $\mathrm{Cd}$-tolerance of perennial ryegrass grown in Cd-contaminated soil, and then improve potential in phytoremediation of cadmium polluted soils.

\section{Materials and Methods}

\subsection{Plant material and culture conditions}

A pot experiment was conducted on a brown clay loam soils (a soil group in Chinese genetic soil classification; named Hapli-Udic Argosols in Chinese soil taxonomy), at College of Resources and Environment, Shandong Agricultural University, Shandong, China. The topsoil $(0-20 \mathrm{~cm})$ had a EC of $197.1 \mu \mathrm{S}$ $\mathrm{cm}^{-1}$ (soil:water ratio 1:5) and contained $14.1 \mathrm{~g} \mathrm{~kg}^{-1}$ organic matter, $0.622 \mathrm{~g} \mathrm{~kg}^{-1}$ total nitrogen, $0.0465 \mathrm{~g}$ $\mathrm{kg}^{-1}$ available nitrogen, $0.0225 \mathrm{~g} \mathrm{~kg}^{-1}$ available phosphorus, and $0.1061 \mathrm{~g} \mathrm{~kg}^{-1}$ available potassium. Soil subsamples were mixed with appropriate amounts of analytical grade solid $\mathrm{CdCl}_{2}$ in order to add $50 \mathrm{mg}$ $\mathrm{kg}^{-1} \mathrm{Cd}$ of soil (oven-dry basis). Basal fertilizers applied were compound fertilizer. The nutrient content of compound fertilizer was $15 \% \mathrm{~N}, 15 \% \mathrm{P}_{2} \mathrm{O}_{5}$, and $15 \% \mathrm{~K}_{2} \mathrm{O}$. The mass of the fertilizer applied in each pot was $667.5 \mathrm{mg}$, and the fertilizer was thoroughly mixed with the soil. Seeds of ryegrass (Lolium perenne L.) were sown on April 27, 2013. Plants were grown in plastic pots (fifty plants per container) with a capacity of $2.5 \mathrm{~kg}$ airdried soil. Pots were arranged in randomized block designs with three replicates. During the growing season, plants were managed under commonly used agronomic and irrigation practices.

The experimental design is given as follows: contrast check (CK): no Cd-contaminated soil, $\mathrm{Cd}$ : 50 $\mathrm{mg} \mathrm{kg}{ }^{-1} \mathrm{Cd}$-contaminated soil, T1: SNP solutions (50 $\mu \mathrm{M}, 100 \mathrm{~mL}$ ) were poured into $50 \mathrm{mg} \mathrm{kg}^{-1} \mathrm{Cd}$-contaminated soil, T2: SNP solutions (100 $\mu \mathrm{M}, 100 \mathrm{~mL})$ were poured into $50 \mathrm{mg} \mathrm{kg}^{-1} \mathrm{Cd}$-contaminated soil, T3: SNP solutions $(300 \mu \mathrm{M}, 100 \mathrm{~mL})$ were poured into $50 \mathrm{mg} \mathrm{kg}^{-1} \mathrm{Cd}$-contaminated soil, T4: SNP solutions $(500 \mu \mathrm{M}, 100 \mathrm{~mL})$ were poured into $50 \mathrm{mg} \mathrm{kg}^{-1}$ Cd-contaminated soil. The SNP solution was poured into soil after a 1-month growth period, and the time interval of twice pour actions was seven days. Three successive harvests were taken. Before the SNP solution was poured into soil, it was stored in the dark. The first was taken after a 2-month growth period, the second after a 3-month growth period, and the third after a 4-month growth period. The ryegrass was clipped with a stainless steel shears at each harvest to leave a $2.5 \mathrm{~cm}$ stubble.

\subsection{Determination of plant growth and root activity}

After 30,60 and 90 days treatment, above-ground fresh and dry biomasses were measured. The samples were separated and oven-dried for $30 \mathrm{~min}$ at $105^{\circ} \mathrm{C}$, then at $70{ }^{\circ} \mathrm{C}$ till the materials reach their constant weights. Root activity was determined by the TTC method according to Zhang and Di (2003). It was expressed as absorbance per unit gram root fresh weight.

\subsection{Determination of chlorophyll content}

The chlorophyll content was determined according to the method of Knudson et al. (1977). Fresh ryegrass leaf $(0.5 \mathrm{~g})$ was extracted in $2 \mathrm{~mL} 95 \%$ ethanol for $24 \mathrm{~h}$ in the dark, and the extracted solution was analyzed. The amounts of chlorophyll a, b and carotenoid were determined using a spectrophotometer (SHIMADZU UV2450 , Kyoto, Japan), by reading the absorbance at 665 , 649 and $470 \mathrm{~nm}$. The chlorophyll content results are expressed as unit's mg per gram-fresh weight $\left(\mathrm{mg} \mathrm{g}^{-1} \mathrm{FW}\right)$. 


\subsection{Determination of $\mathrm{O}_{2}{ }^{\cdot-}$ generation rate and $\mathrm{MDA}$ content}

The production rate of $\mathrm{O}^{\cdot-}$ was measured as described by Elstner and Heupel (1976). Fresh leaves $(0.2 \mathrm{~g})$ were homogenized in $1 \mathrm{~mL}$ of $50 \mathrm{mM}$ phosphate buffer ( $\mathrm{pH} 7.8)$, and the homogenate was centrifuged at $10,0009 \mathrm{~g}$ for $10 \mathrm{~min}$.

Then $0.5 \mathrm{~mL}$ of the supernatant was added to 0.5 $\mathrm{mL} 50 \mathrm{mM}$ phosphate buffer $(\mathrm{pH} 7.8)$ and $0.1 \mathrm{~mL}$ of $10 \mathrm{mM}$ hydroxylamine hydrochloride. After $1 \mathrm{~h}$ reaction at $25{ }^{\circ} \mathrm{C}$, the mixture was added to $1 \mathrm{~mL}$ $17 \mathrm{mM}$ sulfanilamide and $1 \mathrm{~mL} 7 \mathrm{mM}$ a-naphthylamine at $25{ }^{\circ} \mathrm{C}$ for $20 \mathrm{~min}$. The specific absorbance at $530 \mathrm{~nm}$ was determined. Sodium nitrite was used as a standard solution to calculate the production rate of $\mathrm{O}_{2} \cdots$. The level of lipid peroxidation in fresh leaf was measured in terms of malondialdehyde (MDA) concentration by the thiobarbituric acid reaction method (Heath and Packer 1968). MDA concentration was expressed as nmol g-1 $\mathrm{FW}$.

\subsection{Determination of $\mathrm{H}_{2} \mathrm{O}_{2}$ concentration}

Fresh samples $(1.0 \mathrm{~g})$ were homogenized in $2 \mathrm{~mL}$ ice-cold acetone. Titanium reagent $\left(2 \% \mathrm{TiCl}_{2}\right.$ in conc. $\mathrm{HCl}$ ) was added to a known volume of extract supernatant to give a Ti (IV) concentration of $2 \%$. The $\mathrm{Ti}-\mathrm{H}_{2} \mathrm{O}_{2}$ complex, together with unreacted $\mathrm{Ti}$, was then precipitated by adding $0.2 \mathrm{~mL}$ $17 \mathrm{M}$ ammonia solution for each $1 \mathrm{~mL}$ of extract. The precipitate was washed five times with ice acetone by resuspension, drained, and dissolved in 1 $\mathrm{M} \mathrm{H}_{2} \mathrm{SO}_{4}(3 \mathrm{~mL})$. The absorbance of the solution was measured at $410 \mathrm{~nm}$ against blanks, which had been prepared similarly but without plant tissue (Patterson et al., 1984).

\subsection{Determination of antioxidant enzyme activities}

For extraction of antioxidative enzymes, samples were homogenized with $50 \mathrm{mM} \mathrm{Na} \mathrm{HPO}_{4}-\mathrm{NaH}_{2} \mathrm{PO}_{4}$ buffer (pH 7.8) containing $0.2 \mathrm{mM}$ EDTA and $2 \%$ insoluble polyvinylpyrrolidone in a chilled pestle and mortar. The homogenate was centrifuged at $12,000 \times \mathrm{g}$ for $20 \mathrm{~min}$ and the resulted supernatant was used for determination of enzyme activities. The whole extraction procedure was carried out at $4{ }^{\circ} \mathrm{C}$. All spectrophotometric analysis was conducted on a SHIMADZU UV-2450 spectrophotometer (Kyoto, Japan). SOD activity was assayed by measuring its ability to inhibit the photochemical reduction of nitroblue tetrazolium following the method of Stewart and Bewley (1980). CAT activity was measured as the decline in absorbance at $240 \mathrm{~nm}$ due to the decrease of extinction of $\mathrm{H}_{2} \mathrm{O}_{2}$ according to the method of Patra et al. (1978). POD activity was measured by the increase in absorbance at $470 \mathrm{~nm}$ due to guaiacol oxidation (Nickel and Cunningham, 1969).

\subsection{Determination of proline content}

Proline concentration was determined using the method of Bates et al. (1973). After extraction at room temperature with 3\% 5-sulfosalicylic acid solution, the proline content was determined from a standard curve and calculated on fresh weight basis.

\subsection{Determination of soluble protein and ascorbic} acid (AsA) content

Protein was estimated by the method of Bradford (1976). Fresh leaves $(0.5 \mathrm{~g})$ were homogenized in $1 \mathrm{~mL}$ phosphate buffer $(\mathrm{pH} 7.0)$. The crude homogenate was centrifuged at $5,000 \times \mathrm{g}$ for $10 \mathrm{~min}$. 
Half milliliter of freshly prepared trichloroacetic acid (TCA) was added and centrifuged at $8,000 \times \mathrm{g}$ for 15 min. The debris was dissolved in $1 \mathrm{~mL}$ of $0.1 \mathrm{~N} \mathrm{NaOH}$ and $5 \mathrm{~mL}$ Bradford reagent was added.

Absorbance was recorded at $595 \mathrm{~nm}$ using a spectrophotometer (SHIMADZU UV-2450, Japan).

Ascorbic acid (AsA) concentration was measured according to Tonamura (1978). Fresh leaves (0.2 g) were homogenized in ice-cold $2 \mathrm{~mL} 10 \%$ metaphosphoric acid. After centrifugation at $15,000 \times \mathrm{g}$ for 10 $\min$, to $0.5 \mathrm{~mL}$ of supernatant was added $1 \mathrm{~mL}$ citric acid-phosphoric acid buffer ( $\mathrm{pH} 2.3$ ) and $1 \mathrm{~mL} \mathrm{2,}$ 6-dichlorophenol indophenol (30 $\left.\mathrm{mg} \mathrm{L}^{-1}\right)$. After $30 \mathrm{~s}$, the absorbance was determined at $524 \mathrm{~nm}$. AsA concentration was expressed as $\mathrm{mg} \mathrm{g}^{-1} \mathrm{FW}$.

\subsection{Determination cadmium and mineral element concentrations}

The dried tissues were weighed and grinded into powder for the determination of cadmium and mineral element concentrations, which was measured by flame atomic absorbance spectrometry (SHIMADZU AA-6300, Kyoto, Japan) after digested with mixed acid $\left[\mathrm{HNO}_{3}+\mathrm{HClO}_{4}(3: 1, \mathrm{v} / \mathrm{v})\right]$ (Ali et al., 2002).

\subsection{Statistical analysis}

The experiment was a completely random design with three replications. Statistical analyses were carried out by analysis of variance (ANOVA) using SAS software (SAS Institute, Cary NC). Differences between treatments were separated by the least significant difference (LSD) test at a 0.05 probability level.

\section{Results}

\subsection{Plant growth and root activity}

Cd exposure significantly decreased fresh weight, dry weight and root activity of ryegrass plants (Table 1). However, this inhibition was significantly alleviated by 100 and $300 \mu \mathrm{M}$ SNP, especially $300 \mu \mathrm{M}$ SNP. After 30, 60 and 90 days treatment, the fresh weights of T3 treatment were increased by $39.74,59.26$ and $45.11 \%$ compared with Cd-alone treatment, the dry weights were increased by $46.15,56.00$ and $37.04 \%$. But T1 and T4 treatments had no significant effect. Furthermore, the root activity of ryegrass under $\mathrm{Cd}$ stress also increased markedly with the addition of SNP. Taken together, the alleviating effect of SNP was found in a general trend of $\mathrm{T} 3>\mathrm{T} 2>\mathrm{T} 1>\mathrm{T} 4$.

Table 1. Effects of application of different concentrations of NO every seven days on fresh weight, dry weight in leaves and root activity of ryegrass plants grown in Cd-contaminated soil.

\begin{tabular}{cccccccccc}
\hline \multirow{2}{*}{ Treatments } & \multicolumn{3}{c}{ Fresh weight $\left[\mathrm{g}(10 \mathrm{plants})^{-1}\right]$} & \multicolumn{2}{c}{ Dry weight $\left[\mathrm{g}(10 \mathrm{plants})^{-1}\right]$} & \multicolumn{3}{c}{ Root activity $\left[\mu \mathrm{g}(\mathrm{g} \mathrm{h})^{-1} \mathrm{FW}\right]$} \\
\cline { 2 - 8 } & 30 Days & 60 Days & 90 Days & 30 Days & 60 Days & 90 Days & 30 Days & 60 Days & 90 Days \\
\hline CK & $1.09 \pm 0.02 \mathrm{a}$ & $1.88 \pm 0.01 \mathrm{a}$ & $2.22 \pm 0.19 \mathrm{a}$ & $0.21 \pm 0.01 \mathrm{a}$ & $0.39 \pm 0.03 \mathrm{a}$ & $0.39 \pm 0.01 \mathrm{a}$ & $58.83 \pm 2.09 \mathrm{~b}$ & $73.62 \pm 1.99 \mathrm{a}$ & $88.07 \pm 1.16 \mathrm{a}$ \\
$\mathrm{Cd}$ & $0.78 \pm 0.03 \mathrm{~d}$ & $1.08 \pm 0.03 \mathrm{~d}$ & $1.33 \pm 0.02 \mathrm{~d}$ & $0.13 \pm 0.01 \mathrm{~d}$ & $0.25 \pm 0.01 \mathrm{c}$ & $0.27 \pm 0.01 \mathrm{~d}$ & $37.53 \pm 0.96 \mathrm{e}$ & $31.71 \pm 1.63 \mathrm{f}$ & $47.01 \pm 1.94 \mathrm{f}$ \\
$\mathrm{T} 1$ & $0.86 \pm 0.02 \mathrm{c}$ & $1.16 \pm 0.05 \mathrm{~d}$ & $1.42 \pm 0.01 \mathrm{~cd}$ & $0.16 \pm 0.02 \mathrm{c}$ & $0.29 \pm 0.02 \mathrm{c}$ & $0.30 \pm 0.02 \mathrm{c}$ & $43.40 \pm 1.29 \mathrm{~d}$ & $37.13 \pm 1.58 \mathrm{e}$ & $52.71 \pm 1.37 \mathrm{e}$ \\
T2 & $0.98 \pm 0.06 \mathrm{~b}$ & $1.40 \pm 0.07 \mathrm{c}$ & $1.57 \pm 0.02 \mathrm{c}$ & $0.17 \pm 0.02 \mathrm{bc}$ & $0.34 \pm 0.03 \mathrm{~b}$ & $0.34 \pm 0.02 \mathrm{~b}$ & $48.89 \pm 2.29 \mathrm{c}$ & $46.34 \pm 1.22 \mathrm{~d}$ & $68.52 \pm 1.96 \mathrm{c}$ \\
T3 & $1.09 \pm 0.04 \mathrm{a}$ & $1.72 \pm 0.06 \mathrm{~b}$ & $1.93 \pm 0.11 \mathrm{~b}$ & $0.19 \pm 0.01 \mathrm{ab}$ & $0.39 \pm 0.02 \mathrm{a}$ & $0.37 \pm 0.00 \mathrm{a}$ & $61.55 \pm 0.93 \mathrm{a}$ & $65.68 \pm 0.48 \mathrm{~b}$ & $79.92 \pm 1.23 \mathrm{~b}$ \\
T4 & $0.84 \pm 0.04 \mathrm{~cd}$ & $1.16 \pm 0.04 \mathrm{~d}$ & $1.39 \pm 0.01 \mathrm{~b}$ & $0.16 \pm 0.02 \mathrm{~cd}$ & $0.28 \pm 0.02 \mathrm{c}$ & $0.31 \pm 0.01 \mathrm{c}$ & $43.06 \pm 0.80 \mathrm{~d}$ & $57.72 \pm 0.66 \mathrm{c}$ & $64.98 \pm 1.51 \mathrm{~d}$ \\
\hline
\end{tabular}

Note: Values represent the mean \pm S.D. $(n=3)$. Different lowercase letters indicate significant differences at $p<0.05$. 


\subsection{Chlorophyll content}

After 30,60 or 90 days treatment, ryegrass plants under $\mathrm{Cd}$ stress showed a significant decrease in chlorophyll content, chlorophyll a, chlorophyll b and carotenoids (Table 2) concentrations compared to CK. However, When treated with SNP $(50,100$, 300 or $500 \mu \mathrm{M})$ in the presence of Cd-contaminated soil, this inhibition was alleviated, especially 300 $\mu \mathrm{M}$ SNP. In the three consecutive collections of samples, the chlorophyll contents of $\mathrm{T} 3$ were increased by $128.31,76.34$ and $76.78 \%$ compared with $\mathrm{Cd}-$ alone treatment, the chlorophyll a were increased by $110.70,83.07$ and $51.13 \%$, the chlorophyll b were increased by $188.16,66.28$ and $190.73 \%$, the carotenoids were increased by $68.91,92.71$ and $62.77 \%$.

\section{3. $\mathrm{O}_{2}^{-}$-generation rate, $\mathrm{H}_{2} \mathrm{O}_{2}$ and $\mathrm{MDA}$ content}

Compared to the $\mathrm{CK}, \mathrm{Cd}$ treatment significantly increased $\mathrm{O}_{2} \cdot$-generation rate (Table 3 ) by $208.49 \%$ after
30 days, by $334.69 \%$ after 60 days and by $184.25 \%$ after 90 days. The application of exogenous NO could decrease $\mathrm{O}_{2}{ }^{-}$generation rate under Cd stress. The T1, T2, T3 and T4 treatments decreased $\mathrm{O}_{2}{ }^{-}$ generation rate by $15.15,24.21,48.52,37.55 \%$ after 30 days, by $18.37,32.75,56.89,42.04 \%$ after 60 days and by $17.74,32.72,45.74,27.11 \%$ after 90 days than $\mathrm{Cd}$ treatment. The T3 treatment had the lowest $\mathrm{O}_{2}{ }^{-}$-generation rate in leaves of ryegrass plants. Similar findings were found for the $\mathrm{H}_{2} \mathrm{O}_{2}$ content (Table 3).

When plants were subjected to environmental stress, oxidative damage resulted in membrane lipid peroxidation, which could be estimated by MDA content (Table 3). Similar to $\mathrm{O}_{2}{ }^{--}$and $\mathrm{H}_{2} \mathrm{O}_{2}$ change, $\mathrm{Cd}$ treatment significantly increased MDA contents in ryegrass leaves after 30,60 or 90 days, $\mathrm{T} 1, \mathrm{~T} 2, \mathrm{~T} 3$ and $\mathrm{T} 4$ significantly reduced MDA contents in leaves. The T3 treatment had the best effect of alleviation.

Table 2. Effects of application of different concentrations of NO every seven days on the chlorophyll contents ( $\mathrm{mg} \mathrm{g}^{-1} \mathrm{FW}$ ) in leaves of ryegrass plants grown in $\mathrm{Cd}$-contaminated soil.

\begin{tabular}{|c|c|c|c|c|c|c|c|}
\hline \multirow{2}{*}{ Items } & & \multicolumn{6}{|c|}{ Treatments } \\
\hline & & CK & $\mathrm{Cd}$ & $\mathrm{T} 1$ & $\mathrm{~T} 2$ & $\mathrm{~T} 3$ & $\mathrm{~T} 4$ \\
\hline \multirow{3}{*}{$\begin{array}{l}\text { Total Chl } \\
\text { contents }\end{array}$} & 30 days & $2.42 \pm 0.14 \mathrm{a}$ & $0.99 \pm 0.03 \mathrm{e}$ & $1.34 \pm 0.03 \mathrm{~d}$ & $1.78 \pm 0.03 \mathrm{c}$ & $2.27 \pm 0.05 b$ & $1.76 \pm 0.03 \mathrm{c}$ \\
\hline & 60 days & $2.84 \pm 0.06 \mathrm{a}$ & $1.42 \pm 0.07 \mathrm{e}$ & $1.72 \pm 0.06 \mathrm{~d}$ & $2.10 \pm 0.01 \mathrm{c}$ & $2.51 \pm 0.05 b$ & $2.03 \pm 0.09 \mathrm{c}$ \\
\hline & 90 days & $2.74 \pm 0.01 \mathrm{a}$ & $1.39 \pm 0.02 \mathrm{e}$ & $1.62 \pm 0.10 \mathrm{~d}$ & $2.10 \pm 0.08 \mathrm{c}$ & $2.46 \pm 0.01 b$ & $1.66 \pm 0.08 \mathrm{a}$ \\
\hline \multirow{3}{*}{$\begin{array}{c}\text { Chl } a \\
\text { content }\end{array}$} & 30 days & $1.59 \pm 0.05 \mathrm{a}$ & $0.77 \pm 0.03 \mathrm{~d}$ & $1.04 \pm 0.03 \mathrm{c}$ & $1.38 \pm 0.09 b$ & $1.62 \pm 0.03 \mathrm{a}$ & $1.33 \pm 0.03 b$ \\
\hline & 60 days & $1.67 \pm 0.05 \mathrm{a}$ & $0.85 \pm 0.01 \mathrm{~d}$ & $1.05 \pm 0.10 \mathrm{c}$ & $1.26 \pm 0.05 b$ & $1.56 \pm 0.12 \mathrm{a}$ & $1.36 \pm 0.09 \mathrm{~b}$ \\
\hline & 90 days & $1.78 \pm 0.02 \mathrm{a}$ & $1.14 \pm 0.01 \mathrm{~d}$ & $1.29 \pm 0.08 \mathrm{c}$ & $1.59 \pm 0.04 \mathrm{~b}$ & $1.72 \pm 0.03 \mathrm{a}$ & $1.30 \pm 0.10 \mathrm{c}$ \\
\hline \multirow{3}{*}{$\begin{array}{c}\text { Chl } b \\
\text { content }\end{array}$} & 30 days & $0.83 \pm 0.10 \mathrm{a}$ & $0.23 \pm 0.01 \mathrm{e}$ & $0.31 \pm 0.01 \mathrm{de}$ & $0.40 \pm 0.07 \mathrm{~cd}$ & $0.65 \pm 0.05 b$ & $0.42 \pm 0.03 \mathrm{c}$ \\
\hline & 60 days & $1.16 \pm 0.04 \mathrm{a}$ & $0.57 \pm 0.07 \mathrm{e}$ & $0.66 \pm 0.05 \mathrm{de}$ & $0.84 \pm 0.05 \mathrm{c}$ & $0.95 \pm 0.07 \mathrm{~b}$ & $0.67 \pm 0.04 \mathrm{~d}$ \\
\hline & 90 days & $0.96 \pm 0.01 \mathrm{a}$ & $0.26 \pm 0.02 \mathrm{e}$ & $0.33 \pm 0.02 \mathrm{~d}$ & $0.51 \pm 0.04 \mathrm{c}$ & $0.74 \pm 0.02 b$ & $0.36 \pm 0.02 \mathrm{a}$ \\
\hline \multirow{3}{*}{$\begin{array}{c}\text { Carotenoids } \\
\text { content }\end{array}$} & 30 days & $0.39 \pm 0.03 \mathrm{a}$ & $0.24 \pm 0.02 \mathrm{~d}$ & $0.29 \pm 0.04 \mathrm{c}$ & $0.33 \pm 0.03 \mathrm{~d}$ & $0.40 \pm 0.02 \mathrm{a}$ & $0.26 \pm 0.02 \mathrm{ed}$ \\
\hline & 60 days & $0.40 \pm 0.02 \mathrm{a}$ & $0.21 \pm 0.01 \mathrm{~d}$ & $0.26 \pm 0.02 b c$ & $0.31 \pm 0.03 b$ & $0.40 \pm 0.02 \mathrm{a}$ & $0.23 \pm 0.05 \mathrm{~cd}$ \\
\hline & 90 days & $0.39 \pm 0.03 \mathrm{a}$ & $0.22 \pm 0.00 \mathrm{c}$ & $0.25 \pm 0.01 b c$ & $0.29 \pm 0.01 \mathrm{~b}$ & $0.36 \pm 0.04 \mathrm{a}$ & $0.28 \pm 0.02 \mathrm{~b}$ \\
\hline
\end{tabular}

Note: Values represent the mean \pm S.D. $(n=3)$. Different lowercase letters indicate significant differences at $p<0.05$. 
Table 3. Effects of application of different concentrations of $\mathrm{NO}$ every seven days on $\mathrm{O}_{2}{ }_{2}^{--}$generation rate, $\mathrm{H}_{2} \mathrm{O}_{2}$ and MDA content in leaves of ryegrass plants grown in Cd-contaminated soil.

\begin{tabular}{|c|c|c|c|c|c|c|c|c|c|}
\hline \multirow{2}{*}{$\begin{array}{l}\text { Treatment } \\
\mathrm{s}\end{array}$} & \multicolumn{3}{|c|}{$\begin{array}{c}\text { The } \mathrm{O}_{2} \text { generation rate } \\
\left(\mu \mathrm{mol} \mathrm{g} \mathrm{g}^{-1} \mathrm{FW}\right)\end{array}$} & \multicolumn{3}{|c|}{$\begin{array}{l}\mathrm{H}_{2} \mathrm{O}_{2} \text { concentration } \\
\left(\mu \mathrm{mol} \mathrm{g} \mathrm{g}^{-1} \mathrm{FW}\right)\end{array}$} & \multicolumn{3}{|c|}{$\begin{array}{l}\text { MDA concentration } \\
\quad\left(\mathrm{nmol} \mathrm{g}{ }^{-1} \mathrm{FW}\right)\end{array}$} \\
\hline & 30 Days & 60 Days & 90 Days & 30 Days & 60 Days & 90 Days & 30 Days & 60 Days & 90 Days \\
\hline $\mathrm{CK}$ & $15.19 \pm 0.15 f$ & $12.89 \pm 0.52 \mathrm{f}$ & $17.50 \pm 0.36 \mathrm{f}$ & $34.53 \pm 0.98 \mathrm{a}$ & $33.26 \pm 0.25 \mathrm{a}$ & $27.02 \pm 0.51 \mathrm{~b}$ & $15.45 \pm 0.70 \mathrm{~d}$ & $21.91 \pm 0.78 \mathrm{f}$ & $14.11 \pm 0.68 \mathrm{f}$ \\
\hline $\mathrm{Cd}$ & $46.87 \pm 0.80 \mathrm{a}$ & $56.04 \pm 0.06 \mathrm{a}$ & $49.75 \pm 1.28 \mathrm{a}$ & $22.34 \pm 0.48 \mathrm{e}$ & $21.15 \pm 0.10 \mathrm{~d}$ & $16.85 \pm 0.60 \mathrm{e}$ & $26.61 \pm 0.46 \mathrm{a}$ & $37.06 \pm 0.67 \mathrm{a}$ & $28.17 \pm 0.93 \mathrm{a}$ \\
\hline $\mathrm{T} 1$ & $39.77 \pm 0.54 b$ & $45.75 \pm 0.32 b$ & $40.92 \pm 0.63 b$ & $24.60 \pm 0.79 \mathrm{~d}$ & $26.21 \pm 0.40 \mathrm{c}$ & $22.18 \pm 1.11 \mathrm{~d}$ & $23.33 \pm 0.64 b$ & $31.65 \pm 1.22 \mathrm{c}$ & $24.69 \pm 0.43 b$ \\
\hline $\mathrm{T} 2$ & $35.52 \pm 0.80 \mathrm{c}$ & $37.69 \pm 0.42 \mathrm{c}$ & $33.47 \pm 0.65 \mathrm{~d}$ & $27.68 \pm 0.41 \mathrm{c}$ & $27.83 \pm 0.52 \mathrm{c}$ & $27.57 \pm 0.86 b$ & $18.57 \pm 0.76 \mathrm{c}$ & $28.14 \pm 0.74 d$ & $21.17 \pm 0.70 c$ \\
\hline $\mathrm{T} 3$ & $24.13 \pm 0.62 \mathrm{e}$ & $24.16 \pm 0.63 \mathrm{e}$ & $26.99 \pm 1.09 \mathrm{e}$ & $30.24 \pm 0.13 b$ & $31.00 \pm 1.87 \mathrm{~b}$ & $31.61 \pm 1.07 \mathrm{a}$ & $13.13 \pm 0.64 \mathrm{e}$ & $25.03 \pm 0.65 \mathrm{e}$ & $15.55 \pm 0.54 \mathrm{e}$ \\
\hline $\mathrm{T} 4$ & $29.27 \pm 0.34 \mathrm{~d}$ & $32.48 \pm 1.00 \mathrm{~d}$ & $36.26 \pm 1.02 \mathrm{c}$ & $23.73 \pm 0.71 \mathrm{~d}$ & $26.84 \pm 1.04 \mathrm{c}$ & $25.51 \pm 0.46 \mathrm{c}$ & $17.70 \pm 1.01 \mathrm{c}$ & $33.44 \pm 0.54 b$ & $17.21 \pm 0.39 \mathrm{~d}$ \\
\hline
\end{tabular}

Note: Values represent the mean \pm S.D. $(\mathrm{n}=3)$. Different lowercase letters indicate significant differences at $p<0.05$.

Table 4. Effects of application of different concentrations of NO every seven days on SOD, POD and CAT content in leaves of ryegrass plants grown in Cd-contaminated soil.

\begin{tabular}{|c|c|c|c|c|c|c|c|c|c|}
\hline \multirow{2}{*}{ Treatments } & \multicolumn{3}{|c|}{$\begin{array}{l}\text { SOD activity } \\
\left(\mathrm{U} \mathrm{g}^{-1} \mathrm{FW}\right)\end{array}$} & \multicolumn{3}{|c|}{$\begin{array}{c}\text { POD activity } \\
\left(\mathrm{U} \mathrm{mg}^{-1} \mathrm{~min}^{-1} \mathrm{FW}\right)\end{array}$} & \multicolumn{3}{|c|}{$\begin{array}{c}\text { CAT activity } \\
\left(\mu \mathrm{mol} \mathrm{H} \mathrm{O}_{2} \mathrm{mg}^{-1} \mathrm{~min}^{-1} \mathrm{FW}\right)\end{array}$} \\
\hline & 30 Days & 60 Days & 90 Days & 30 Days & 60 Days & 90 Days & 30 Days & 60 Days & 90 Days \\
\hline $\mathrm{CK}$ & $112.50 \pm 3.40 \mathrm{a}$ & $97.52 \pm 1.56 \mathrm{a}$ & $168.59 \pm 3.76 \mathrm{a}$ & $97.56 \pm 3.26 \mathrm{a}$ & $203.76 \pm 1.42 b$ & $228.85 \pm 1.50 \mathrm{a}$ & $8.13 \pm 0.12 \mathrm{a}$ & $8.28 \pm 0.02 \mathrm{a}$ & $8.45 \pm 0.07 \mathrm{a}$ \\
\hline $\mathrm{Cd}$ & $59.64 \pm 1.18 \mathrm{e}$ & $44.29 \pm 3.70 \mathrm{f}$ & $96.63 \pm 1.87 \mathrm{e}$ & $51.68 \pm 4.21 \mathrm{e}$ & $154.26 \pm 0.60 \mathrm{e}$ & $161.98 \pm 1.77 \mathrm{f}$ & $5.93 \pm 0.08 \mathrm{e}$ & $6.30 \pm 0.06 \mathrm{e}$ & $5.76 \pm 0.05 f$ \\
\hline $\mathrm{T} 1$ & $64.62 \pm 0.42 \mathrm{~d}$ & $54.76 \pm 1.92 \mathrm{e}$ & $106.14 \pm 2.33 \mathrm{~d}$ & $64.59 \pm 1.78 \mathrm{~d}$ & $166.85 \pm 1.05 \mathrm{~d}$ & $180.86 \pm 1.17 \mathrm{e}$ & $6.22 \pm 0.05 \mathrm{~d}$ & $6.58 \pm 0.12 \mathrm{~d}$ & $6.24 \pm 0.10 \mathrm{e}$ \\
\hline $\mathrm{T} 2$ & $74.03 \pm 1.25 \mathrm{c}$ & $66.10 \pm 1.90 \mathrm{c}$ & $112.77 \pm 2.39 \mathrm{c}$ & $76.44 \pm 2.00 \mathrm{c}$ & $197.37 \pm 1.62 \mathrm{c}$ & $209.92 \pm 0.97 \mathrm{c}$ & $6.79 \pm 0.14 \mathrm{c}$ & $7.27 \pm 0.02 \mathrm{c}$ & $7.34 \pm 0.12 \mathrm{c}$ \\
\hline $\mathrm{T} 3$ & $86.09 \pm 1.93 b$ & $83.22 \pm 2.30 \mathrm{~b}$ & $138.43 \pm 3.44 b$ & $90.28 \pm 1.53 b$ & $246.80 \pm 2.19 \mathrm{a}$ & $221.66 \pm 1.35 b$ & $8.13 \pm 0.09 \mathrm{a}$ & $7.55 \pm 0.13 b$ & $8.18 \pm 0.08 b$ \\
\hline $\mathrm{T} 4$ & $68.06 \pm 2.04 \mathrm{a}$ & $59.60 \pm 2.78 \mathrm{~d}$ & $111.15 \pm 3.04 \mathrm{~cd}$ & $65.17 \pm 1.03 \mathrm{~d}$ & $203.53 \pm 1.91 \mathrm{~b}$ & $185.68 \pm 2.43 \mathrm{~d}$ & $7.54 \pm 0.02 b$ & $6.18 \pm 0.06 \mathrm{e}$ & $6.95 \pm 0.10 \mathrm{~d}$ \\
\hline
\end{tabular}

Note: Values represent the mean \pm S.D. $(n=3)$. Different lowercase letters indicate significant differences at $\mathrm{p}<0.05$.

\subsection{Antioxidant enzymes}

As shown in Table 4, Superoxide dismutase activity decreased markedly under $\mathrm{Cd}$ stress in leaves of ryegrass plants. After 30,60 or 90 days treatment, $\mathrm{Cd}$ treatment significantly decreased SOD activity by $46.99,54.59$ and $42.69 \%$ in leaves. The T1, T2, T3 and T4 treatments increased SOD activity by 8.35 , $24.13,44.36,14.13 \%$ after 30 days, by 23.64, 49.26, $87.90,34.57 \%$ after 60 days and by $9.84,16.71$, 43.27, $15.03 \%$ after 90 days than $\mathrm{Cd}$ treatment. It was similar in activities of POD and CAT. All of antioxidant enzyme activities were found to be in a general trend of $\mathrm{CK}>\mathrm{T} 3>\mathrm{T} 2>\mathrm{T} 1$, or $\mathrm{T} 4>\mathrm{Cd}$.

\subsection{Proline content}

To investigate the contributions of non enzymatic antioxidants to the response of ryegrass to $\mathrm{Cd}$ toxicity, proline content was examined. After 30, 60 and 90 days treatment, Proline content in leaves of ryegrass plants (Figure 1) decreased by 50.57, 34.82 and $45.51 \%$ in response to $\mathrm{Cd}$ application. The T1, T2, T4 and especially T3 treatments significantly increased proline content in Cd-treated plants. Compared to the $\mathrm{Cd}$ alone treatment, after 30, 60 and 90 days treatment, T3 treatment increased proline content by $105.63,54.49$ and $106.52 \%$. 


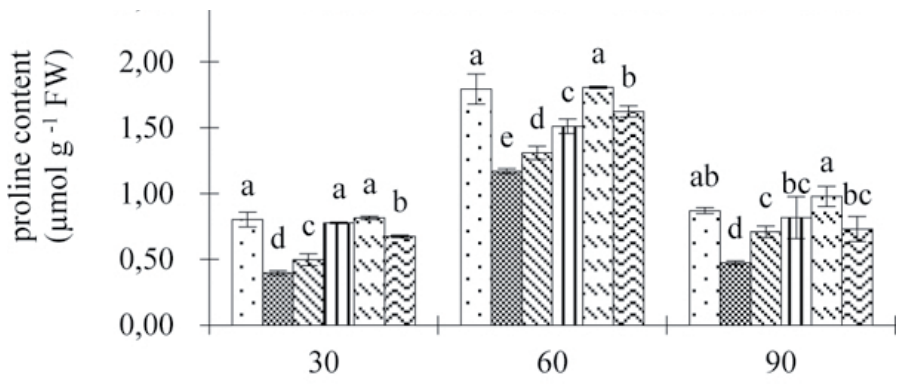

Time (Days)

Figure 1. Effects of application of different concentrations of NO every seven days on proline content in leaves of ryegrass plants grown in Cd-contaminated soil. Values are the mean of three replicates. Values are the mean of three replicates. Each replicate has 50 plants. Bars with different letters are significantly different at $P<0.05$.

Table 5. Effects of application of different concentrations of $\mathrm{NO}$ every seven days on soluble protein and AsA content in leaves of ryegrass plants grown in Cd-contaminated soil.

\begin{tabular}{|c|c|c|c|c|c|c|}
\hline \multirow[t]{2}{*}{ Treatments } & \multicolumn{3}{|c|}{$\begin{array}{c}\text { Soluble protein content } \\
\left(\mathrm{mg} \mathrm{g}^{-1} \mathrm{FW}\right)\end{array}$} & \multicolumn{3}{|c|}{$\begin{array}{c}\text { AsA content } \\
\left(\mathrm{nmol} \mathrm{g}{ }^{-1} \mathrm{FW}\right)\end{array}$} \\
\hline & 30 Days & 60 Days & 90 Days & 30 Days & 60 Days & 90 Days \\
\hline CK & $21.13 \pm 0.48 \mathrm{~b}$ & $29.33 \pm 0.41 \mathrm{~b}$ & $23.09 \pm 0.38 \mathrm{c}$ & $22.95 \pm 0.14 \mathrm{a}$ & $12.45 \pm 0.37 \mathrm{a}$ & $12.82 \pm 0.19 \mathrm{a}$ \\
\hline $\mathrm{Cd}$ & $12.52 \pm 0.58 \mathrm{e}$ & $20.85 \pm 0.47 \mathrm{e}$ & $15.41 \pm 0.08 \mathrm{f}$ & $13.50 \pm 0.75 \mathrm{e}$ & $3.81 \pm 0.05 \mathrm{f}$ & $6.21 \pm 0.12 \mathrm{f}$ \\
\hline $\mathrm{T} 1$ & $16.23 \pm 0.71 \mathrm{~d}$ & $25.28 \pm 0.56 \mathrm{~d}$ & $17.68 \pm 0.94 \mathrm{e}$ & $14.86 \pm 0.30 \mathrm{~d}$ & $4.47 \pm 0.06 \mathrm{e}$ & $7.10 \pm 0.03 \mathrm{e}$ \\
\hline $\mathrm{T} 2$ & $18.16 \pm 0.50 \mathrm{c}$ & $27.69 \pm 0.72 \mathrm{c}$ & $21.05 \pm 0.95 \mathrm{~d}$ & $16.43 \pm 0.35 \mathrm{c}$ & $6.90 \pm 0.04 \mathrm{c}$ & $7.99 \pm 0.06 \mathrm{~d}$ \\
\hline $\mathrm{T} 3$ & $22.90 \pm 1.45 \mathrm{a}$ & $32.75 \pm 0.90 \mathrm{a}$ & $32.09 \pm 0.05 \mathrm{a}$ & $17.95 \pm 0.35 b$ & $8.81 \pm 0.05 b$ & $10.55 \pm 0.19 \mathrm{~b}$ \\
\hline $\mathrm{T} 4$ & $18.68 \pm 0.47 \mathrm{c}$ & $28.49 \pm 0.34 b c$ & $25.81 \pm 0.59 \mathrm{~b}$ & $16.45 \pm 0.69 \mathrm{c}$ & $5.85 \pm 0.13 \mathrm{~d}$ & $8.70 \pm 0.10 \mathrm{c}$ \\
\hline
\end{tabular}

Note: Values represent the mean \pm S.D. $(n=3)$. Different lowercase letters indicate significant differences at $p<0.05$.

\subsection{Soluble protein and AsA content}

Cd treatment decreased soluble protein content and AsA content in leaves of ryegrass plants dramatically (Table 5). However, these inhibitions were significantly alleviated by $50,100,300$ and $500 \mu \mathrm{M}$ SNP, especially $300 \mu \mathrm{M}$ SNP. Compared with $\mathrm{Cd}$ treatment, the soluble protein and AsA content of T3 treatment were increased by $82.95,32.96 \%$ after 30 days, by $57.08,131.03 \%$ after 60 days and by $108.31,70.00 \%$ after 90 days.

\subsection{Cd concentrations}

Cadmium accumulation was significantly higher in roots than in shoots (Table 6) and Cd concentrations in shoots and roots of Cd-treated plants were accumulated at a high level. However, after 30, 60 and 90 days treatment, $\mathrm{Cd}$ accumulation was decreased not only in shoots but also in roots with addition of SNP in Cd-contaminated soil, and $\mathrm{T} 3$ treatment had the best effect on inhibiting $\mathrm{Cd}$ accumulation in shoots and roots of ryegrass plants. 
Table 6. Effects of application of different concentrations of NO every seven days on Cd content ( $\left.\mathrm{mg} \mathrm{kg}^{-1} \mathrm{DW}\right)$ in shoots and roots of ryegrass plants grown in Cd-contaminated soil

\begin{tabular}{ccccccc}
\hline \multirow{2}{*}{ Treatments } & \multicolumn{2}{c}{ 30 Days } & \multicolumn{2}{c}{60 Days } & \multicolumn{2}{c}{90 Days } \\
\cline { 2 - 7 } & Shoots & Roots & Shoots & Roots & Shoots & Roots \\
\hline CK & $0.00 \pm 0.00 \mathrm{e}$ & $0.00 \pm 0.00 \mathrm{e}$ & $0.00 \pm 0.00 \mathrm{e}$ & $0.00 \pm 0.00 \mathrm{f}$ & $0.00 \pm 0.00 \mathrm{f}$ & $0.00 \pm 0.00 \mathrm{e}$ \\
Cd & $142.40 \pm 5.14 \mathrm{a}$ & $1039.51 \pm 33.30 \mathrm{a}$ & $117.92 \pm 4.41 \mathrm{a}$ & $871.23 \pm 8.49 \mathrm{a}$ & $131.09 \pm 4.87 \mathrm{a}$ & $819.38 \pm 4.97 \mathrm{a}$ \\
T1 & $132.51 \pm 1.26 \mathrm{~b}$ & $898.82 \pm 25.93 \mathrm{~b}$ & $108.25 \pm 2.36 \mathrm{~b}$ & $734.01 \pm 8.59 \mathrm{c}$ & $113.15 \pm 3.94 \mathrm{c}$ & $760.77 \pm 25.96 \mathrm{~b}$ \\
T2 & $121.78 \pm 3.22 \mathrm{c}$ & $835.16 \pm 22.55 \mathrm{c}$ & $102.70 \pm 2.65 \mathrm{c}$ & $560.30 \pm 20.83 \mathrm{~d}$ & $101.54 \pm 4.42 \mathrm{~d}$ & $666.83 \pm 3.18 \mathrm{c}$ \\
T3 & $114.57 \pm 1.41 \mathrm{~d}$ & $750.43 \pm 7.58 \mathrm{~d}$ & $95.26 \pm 1.53 \mathrm{~d}$ & $485.58 \pm 13.84 \mathrm{e}$ & $87.44 \pm 2.26 \mathrm{e}$ & $579.09 \pm 18.93 \mathrm{~d}$ \\
T4 & $144.39 \pm 7.28 \mathrm{a}$ & $851.22 \pm 34.65 \mathrm{c}$ & $103.28 \pm 0.78 \mathrm{c}$ & $771.46 \pm 20.23 \mathrm{~b}$ & $124.18 \pm 4.48 \mathrm{~b}$ & $818.92 \pm 11.11 \mathrm{a}$ \\
\hline
\end{tabular}

Note: Values represent the mean \pm S.D. $(\mathrm{n}=3)$. Different lowercase letters indicate significant differences at $p<0.05$.

Table 7. Effects of application of different concentrations of NO every seven days on mineral content $\left(\mathrm{g} \mathrm{kg}^{-1} \mathrm{DW}\right)$ in leaves and roots of ryegrass plants grown in Cd-contaminated soil.

\begin{tabular}{|c|c|c|c|c|c|c|c|}
\hline \multirow{2}{*}{ Items } & & \multicolumn{6}{|c|}{ Treatments } \\
\hline & & CK & $\mathrm{Cd}$ & $\mathrm{T} 1$ & $\mathrm{~T} 2$ & $\mathrm{~T} 3$ & $\mathrm{~T} 4$ \\
\hline \multicolumn{8}{|l|}{30 days } \\
\hline \multirow[b]{2}{*}{$\mathrm{Ca}$} & Shoots & $0.19 \mathrm{ab}$ & $0.13 \mathrm{~d}$ & $0.16 \mathrm{c}$ & $0.17 \mathrm{~b}$ & $0.19 \mathrm{a}$ & $0.16 \mathrm{c}$ \\
\hline & Roots & $0.16 \mathrm{a}$ & $0.09 \mathrm{~b}$ & $0.10 \mathrm{~b}$ & $0.15 \mathrm{a}$ & $0.16 \mathrm{a}$ & $0.10 \mathrm{~b}$ \\
\hline \multirow{2}{*}{$\mathrm{Fe}$} & Shoots & $0.96 \mathrm{a}$ & $0.36 \mathrm{e}$ & $0.39 \mathrm{~d}$ & $0.45 \mathrm{c}$ & $0.61 \mathrm{~b}$ & $0.34 \mathrm{e}$ \\
\hline & Roots & $1.73 \mathrm{a}$ & $0.74 \mathrm{e}$ & $0.91 \mathrm{~d}$ & $1.03 \mathrm{c}$ & $1.52 \mathrm{~b}$ & $0.98 \mathrm{~cd}$ \\
\hline \multirow{2}{*}{$\mathrm{Mg}$} & Shoots & $3.11 \mathrm{c}$ & $2.67 \mathrm{e}$ & $2.86 \mathrm{~d}$ & $3.30 \mathrm{~b}$ & $3.56 \mathrm{a}$ & $2.79 \mathrm{~d}$ \\
\hline & Roots & $5.15 \mathrm{a}$ & $2.57 \mathrm{~d}$ & $2.78 \mathrm{~d}$ & $3.24 \mathrm{c}$ & $4.42 b$ & $3.25 \mathrm{c}$ \\
\hline \multirow[b]{2}{*}{$\mathrm{Zn}$} & Shoots & $3.56 \mathrm{a}$ & $2.57 \mathrm{e}$ & $2.85 \mathrm{c}$ & $3.04 \mathrm{~b}$ & $3.59 \mathrm{a}$ & $2.74 \mathrm{~d}$ \\
\hline & Roots & $6.01 \mathrm{a}$ & $3.64 \mathrm{e}$ & $3.97 \mathrm{~d}$ & $4.35 \mathrm{c}$ & $5.39 \mathrm{~b}$ & $3.93 \mathrm{~d}$ \\
\hline \multicolumn{8}{|l|}{60 days } \\
\hline \multirow[b]{2}{*}{$\mathrm{Ca}$} & Shoots & $0.33 \mathrm{a}$ & $0.16 \mathrm{e}$ & $0.19 \mathrm{~d}$ & $0.21 \mathrm{c}$ & $0.28 b$ & $0.22 \mathrm{c}$ \\
\hline & Roots & $0.15 \mathrm{a}$ & $0.05 \mathrm{c}$ & $0.10 \mathrm{~b}$ & $0.11 \mathrm{~b}$ & $0.14 \mathrm{a}$ & $0.10 \mathrm{~b}$ \\
\hline \multirow{2}{*}{$\mathrm{Fe}$} & Shoots & $0.46 \mathrm{a}$ & $0.23 \mathrm{e}$ & $0.26 \mathrm{~d}$ & $0.32 \mathrm{c}$ & $0.39 \mathrm{~b}$ & $0.25 \mathrm{~d}$ \\
\hline & Roots & $1.12 \mathrm{a}$ & $0.68 \mathrm{~d}$ & $0.81 \mathrm{c}$ & $0.90 \mathrm{~b}$ & $1.07 \mathrm{a}$ & $0.85 b c$ \\
\hline \multirow{2}{*}{$\mathrm{Mg}$} & Shoots & $3.02 \mathrm{a}$ & $1.77 \mathrm{f}$ & $1.93 \mathrm{e}$ & $2.14 \mathrm{c}$ & $2.41 \mathrm{~b}$ & $2.01 \mathrm{a}$ \\
\hline & Roots & $2.84 \mathrm{a}$ & $1.98 \mathrm{f}$ & $2.17 \mathrm{e}$ & $2.37 \mathrm{c}$ & $2.54 \mathrm{~b}$ & $2.26 \mathrm{~d}$ \\
\hline \multirow[b]{2}{*}{$\mathrm{Zn}$} & Shoots & $4.28 \mathrm{a}$ & $2.22 \mathrm{f}$ & $2.46 \mathrm{~d}$ & $2.96 \mathrm{c}$ & $3.45 b$ & $2.33 \mathrm{e}$ \\
\hline & Roots & $5.20 \mathrm{~b}$ & $3.98 \mathrm{e}$ & $4.15 \mathrm{~d}$ & $4.57 \mathrm{c}$ & $5.39 \mathrm{a}$ & $4.22 \mathrm{~d}$ \\
\hline \multicolumn{8}{|l|}{90 days } \\
\hline \multirow{2}{*}{$\mathrm{Ca}$} & Shoots & $0.10 \mathrm{a}$ & $0.04 \mathrm{~d}$ & $0.05 \mathrm{~cd}$ & $0.06 \mathrm{bc}$ & $0.10 \mathrm{a}$ & $0.07 \mathrm{~b}$ \\
\hline & Roots & $0.06 \mathrm{~b}$ & $0.04 d$ & $0.05 \mathrm{~cd}$ & $0.06 \mathrm{bc}$ & $0.07 \mathrm{a}$ & $0.06 \mathrm{bc}$ \\
\hline \multirow{2}{*}{$\mathrm{Fe}$} & Shoots & $0.43 \mathrm{a}$ & $0.22 \mathrm{~d}$ & $0.32 \mathrm{c}$ & $0.37 \mathrm{~b}$ & $0.43 \mathrm{a}$ & $0.36 \mathrm{~b}$ \\
\hline & Roots & $1.50 \mathrm{a}$ & $0.64 \mathrm{e}$ & $1.06 \mathrm{~d}$ & $1.14 \mathrm{c}$ & $1.33 \mathrm{~b}$ & $1.10 \mathrm{~cd}$ \\
\hline \multirow{2}{*}{$\mathrm{Mg}$} & Shoots & $2.75 \mathrm{a}$ & $2.06 \mathrm{c}$ & $2.27 \mathrm{~b}$ & $2.37 \mathrm{~b}$ & $2.65 \mathrm{a}$ & $2.02 \mathrm{c}$ \\
\hline & Roots & $3.68 \mathrm{a}$ & $2.56 \mathrm{e}$ & $2.86 \mathrm{~d}$ & $3.25 \mathrm{c}$ & $3.53 \mathrm{~b}$ & $2.92 \mathrm{a}$ \\
\hline \multirow{2}{*}{$\mathrm{Zn}$} & Shoots & $4.35 \mathrm{a}$ & $2.63 \mathrm{e}$ & $3.06 \mathrm{~d}$ & $3.43 \mathrm{c}$ & $3.70 \mathrm{~b}$ & $3.36 \mathrm{c}$ \\
\hline & Roots & $4.00 \mathrm{a}$ & $2.77 \mathrm{e}$ & $2.97 \mathrm{~d}$ & $3.26 \mathrm{c}$ & $3.71 \mathrm{~b}$ & $3.18 \mathrm{c}$ \\
\hline
\end{tabular}

Note: Values represent the mean \pm S.D. $(\mathrm{n}=3)$. Different lowercase letters indicate significant differences at $p<0.05$. 


\section{8. $\mathrm{Ca}, \mathrm{Fe}, \mathrm{Mg}$ and $\mathrm{Zn}$ contents}

Table 7 showed $\mathrm{Ca}, \mathrm{Fe}, \mathrm{Mg}$ and $\mathrm{Zn}$ contents in shoots and roots of ryegrass plants. Compared with $\mathrm{CK}, \mathrm{Cd}$ treatment significantly decreased $\mathrm{Ca}, \mathrm{Fe}, \mathrm{Mg}$ and $\mathrm{Zn}$ contents in shoots and in roots. However, the application of SNP increased $\mathrm{Ca}, \mathrm{Fe}, \mathrm{Mg}$ and $\mathrm{Zn}$ contents in shoots and in roots, and $300 \mu \mathrm{M}$ SNP had the most obvious promotion on mineral concentrations. Taken together, the alleviating effect of SNP was found in a general trend of $\mathrm{T} 3>\mathrm{T} 2>\mathrm{T} 4>\mathrm{T} 1$.

\section{Discussion}

In the recent decades, an increasing number of studies have reported the effect of SNP on alleviating heavy metal toxicity in plants (Chen et al., 2010; Xu et al., 2010; Wang et al., 2013). In the present experiment, we investigated the effects of application of different concentrations of $\mathrm{NO}$ on ryegrass plants under $\mathrm{Cd}$ stress grown in $50 \mathrm{mg} \mathrm{kg}^{-1} \mathrm{Cd}$-contaminated soil and analyzed the possible roles of exogenous $\mathrm{NO}$ in mitigating Cd toxicity. After 30, 60 and 90 days treatment, compared to $\mathrm{CK}$, the growth of ryegrass plants was inhibited significantly under $\mathrm{Cd}$ stress (Table 1). The inhibition of growth in ryegrass might be resulted from Cd-induced alteration of fundamental metabolic process, photosynthesis and uptake of nutrient elements. Cadmium is phytotoxic, as it can interfere with photosynthetic and respiratory activities, mineral nutrition, enzymatic activities, membrane functions, and hormone balance. Cd may lead to changes in plant nutrient concentration and composition. However, the application of SNP had a promoting effect on plant growth under Cd stress (Table 1). The mitigation effect may be due to the fact that the optimum concentration of NO can act directly on the cell wall components and plasma membrane bilayers, resulting in enhancing membrane fluidity and promoting cell expansion and growth (Xiong et al., 2009; Wang et al., 2013).

A notable reduction of chlorophyll concentration was detected under $\mathrm{Cd}$ stress in ryegrass leaves (Table 2). Chen et al. (2008) suggested that decreasing leaf chlorophyll content was one of the most general toxicity effects of $\mathrm{Cd}$ on plants, which was confirmed again in the present study. The decrease in chlorophyll content in Cd-affected ryegrass plants might be attributed to the possible oxidation of chlorophyll and the damaged ultrastructure of chloroplasts under Cd stress (Chen et al., 2010). However, the addition of SNP increased chlorophyll content dramatically, and the best of alleviating effect was T3 treatment. In this experiment, the application of SNP and especially $300 \mu \mathrm{M}$ SNP significantly increased $\mathrm{Fe}$ concentration in both the shoots and roots of ryegrass and then increased chlorophyll content. In addition, SNP may effectively reduce the level of ROS generated during stress resulting in alleviating the oxidative negative effects of ROS on growth and chlorophyll content.

It has been shown that $\mathrm{Cd}$ leads to an increased production of $\mathrm{H}_{2} \mathrm{O}_{2}$ and induces lipid peroxidation (Tamás et al., 2015) in plants. MDA content was measured as an index of lipid peroxidation. In our experiment, we investigated the involvement of these molecules. The markedly increases in contents of $\mathrm{H}_{2} \mathrm{O}_{2}, \mathrm{O}_{2}{ }^{-}$and MDA under Cd stress were observed (Table 3). Similar results have been observed in ryegrass and lettuce (Wang et al., 2013; Xu et al., 2014).

It is also widely agreed that plants resist the stress induced production of ROS by increasing components of their intrinsic defense system including altered activities of enzyme proteins (Manai et al., 2014). SOD constitutes the first line of defense against ROS, which is crucial for the removal of $\mathrm{O}_{2}^{-}$- in the compartments where $\mathrm{O}_{2}{ }^{*-}$ radicals formed. POD catalyses $\mathrm{H}_{2} \mathrm{O}_{2}$-dependent oxidation of substrate, while CAT and APX eliminate $\mathrm{H}_{2} \mathrm{O}_{2}$ by breaking it down directly to form 
water and oxygen. In the present study, Cd stress led to decreases in activities of SOD, POD and CAT (Table 4). This result was in agreement with Tamás et al. (2015) who reported that exposure to Cd stress significantly decreased activities of SOD, CAT and POD. However, exogenous NO improved activities of SOD, POD and CAT (Table 4). The similar results have been observed in rice under Cd stress (Xiong et al., 2009). SNP -induced antioxidant enzyme activities increase indicated that they can play a critical role in modulating the cell redox balance, thereby protecting plants against oxidative damage. Osmotic adjustment is the main part of the physiological machinery by which plants respond to heavy metals toxicity. Proline plays important roles on clearance of ROS and adjustment of osmotic substances (Dong et al., 2014). There are recent reports suggesting that NO improve Cd tolerance in M. truncatula roots (Xu et al., 2010) and rice seedlings (He et al., 2014) by an increase in the production of proline. As shown in Figure 1, proline content decreased in ryegrass plants grown with $\mathrm{Cd}$ stress alone. However, the addition of SNP and especially $300 \mu \mathrm{M}$ SNP significantly increased proline content in Cd-treated plants. In fact, proline has multiple functions under stressful conditions, such as osmotic pressure regulation, protection of membrane integrity, stabilization of enzymes/proteins, maintaining appropriate NADP+/NADPH ratios, and scavenger of free radicals (Idrees et al., 2012).

Results of our study showed that Cd decreased the content of soluble protein in ryegrass plants significantly, and treatments with SNP increased the level of soluble protein (Table 5). This study coincided with Ericson and Alfinito (1984) who reported that abiotic stress may inhibit a synthesis of some proteins and promote others with a general trend of decline in the overall content. However, SNP supplementation improved Cd-decreased soluble protein content (Table 5). It is likely that nitric oxide (NO) could alleviate the heavy metal-induced phytotoxicity and had very important role in protein nitration (Saxena and Shekhawat, 2013). Ascorbic acid (ASA) is the most abundant, powerful and water soluble antioxidant acts preventing or at least alleviating deleterious effects caused by ROS in plants, thus providing membrane protection. However, in this present study, $\mathrm{Cd}$ decreased the content of ASA in ryegrass plants significantly, and the treatments with SNP increased the ASA content (Table 5). This suggested SNP mediated the enhancement of AsA levels in ryegrass plants. Moreover, ASA can participate in detoxifying ROS directly or through certain enzymes restricting lipid peroxidation and oxidative stress, and then improved the tolerance of $\mathrm{Cd}$ toxicity.

Tight control of $\mathrm{Cd}$ in sensitive tissues may act as the first line of defense in plants to counteract $\mathrm{Cd}$ toxicity. In plants, the root is in direct contact with $\mathrm{Cd}$ and the cell walls of roots play a significant role in heavy metal tolerance. A principal defense step to counteract $\mathrm{Cd}$ toxicity in plants is to prevent $\mathrm{Cd}$ accumulation in shoot tissues. It was documented by Xiong et al. (2009) that exogenous NO alleviated Cd toxicity in rice by increasing pectin and hemicellulose contents in root cell walls, increasing $\mathrm{Cd}$ deposition in root cells and decreasing $\mathrm{Cd}$ accumulation in soluble fractions of leaves. Previous reports also indicated SNP supplementation at low concentrations reduced the transport of $\mathrm{Cd}$ from roots to shoots in perennial ryegrass (Wang et al., 2013). In this work, increased uptake of $\mathrm{Cd}$ in both roots and shoots as compared to control is noted in Cd-treated plants. And exposure of plants to $\mathrm{Cd}$ stress resulted in that $\mathrm{Cd}$ accumulated at higher concentrations in roots than in shoots (Table 6). This suggests that the ability of ryegrass plants to prevent excessive accumulation of $\mathrm{Cd}$ in shoots in the presence of Cd stress may at least partially account for the tolerance to $\mathrm{Cd}$. However, addition of SNP in the growth medium lowered the accumulation of $\mathrm{Cd}$ 
and the root-to-shoot translocation of $\mathrm{Cd}$ in $\mathrm{Cd}+\mathrm{SNP}$ treatment than that in plants grown under $\mathrm{Cd}$ alone (Table 6). This result is consistent with the findings of Wang et al. (2013).

Cadmium toxicity has been related to interactions between uptake and translocation of mineral nutrients in plants. In our study, Cd toxicity led to a deficiency of $\mathrm{Ca}, \mathrm{Mg}, \mathrm{Fe}$ and $\mathrm{Zn}$ in the ryegrass plants (Table 7), disturbed intracellular ion homeostasis, and then result in growth inhibition. Similar results were reported in our previous study (Wang et al., 2013). These results demonstrated that $\mathrm{Cd}$ interfered with nutrient uptake and with nutrient distribution into the different plant parts. However, the SNP treatment was found to promote the uptake of these mineral elements (Table 7). This could be explained by SNP increasing the activity of $\mathrm{H}^{+}$-ATPase, which plays an important role in the transport of multiple ions. Moreover, $\mathrm{Ca}$ provides intermolecular linkages and is thought to play a crucial role in the stabilization of cell wall and membrane. Neill et al. (2002) demonstrated that NO could interact with $\mathrm{Ca}^{2+}$ channel in plants. In addition, the addition of SNP can promote the uptake of $\mathrm{Ca}$ in plants under Cd stress. For these reasons, the uptake of $\mathrm{Mg}, \mathrm{Fe}$ and $\mathrm{Zn}$ from medium to plant was promoted by the addition of SNP. These results indicated that SNP could ameliorate ion equilibrium in ryegrass cells under $\mathrm{Cd}$ stress and promoted plant growth.

\section{Conclusion}

This study demonstrated that, Cd stress depressed plant growth, reflected by oxidative stress and the inhibition of chlorophyll synthesis and nutrient elements uptake. However, application of SNP at a proper concentration could significantly alleviate the effect of $\mathrm{Cd}$ toxicity in ryegrass plants exposed to $\mathrm{Cd}$ stress. The potential mechanisms include: (1) promoted chlorophyll synthesis; (2) induced a better antioxidant system in plants; (3) reduced Cd uptake, inhibited $\mathrm{Cd}$ transport from roots to shoots and enhanced the absorption of nutrient elements.

And $300 \mu \mathrm{M}$ SNP had the best of alleviating effect against $\mathrm{Cd}$ toxicity, while high concentrations of SNP had no significant effect. Our results may have a potential value on repair of heavy metal contaminated areas, but further study is required.

\section{Acknowledgement}

This research work was financially supported by a Project of Shandong Province Higher Educational Science and Technology Program (J14LF08) and the Shandong Provincial Natural Science Foundation of China (ZR2013CM003). Special acknowledgements are given to the editors and reviewers.

\section{Reference}

Ali, N.A., Bernal, M.P., Ater, M. 2002. Tolerance and bioaccumulation of copper in Phragmites australis and Zea mays. Plant Soil. 239, 103-111.

Arienzo, M., Adamo, P., Cozzolino, V. 2004. The potential of Lolium perenne for revegetation of contaminated soil from a metallurgical site. Sci. Total Environ. 319, 13-25.

Bates, L.S., Waldern, S.P., Teare, I.D. 1973. Rapid determination of free proline for water-stress studies. Plant Soil. 39, 205-207.

Bradford, M.M. 1976. A rapid and sensitive method for the quantitation of microgram quantities of protein utilizating the principle of protein dyes binding. Anal. Biochem. 72, 248-254.

Chen, F., Wang, F., Sun, H.Y., Cai, Y., Miao, W.H., Zhang, G.P., Vincze, E., Wu, F.B. 2010. Genotypedependent effect of exogenous nitric oxide on Cd-induced changes in antioxidative metabolism, 
ultrastructure, and photosynthetic performance in barley seedlings (Hordeum vulgare). J. Plant Growth Regul. 29, 394-408.

Chen, F., Wang, F., Zhang, G.P., Wu, F.B. 2008. Identification of barley varieties tolerant to cadmium toxicity. Biol. Trace Elem. Res. 121, 171-179.

Chen, G.C., Liu, Y.Q., Wang, R.M., Zhang, J.F., Owens, G. 2013. Cadmium adsorption by willow root: the role of cell walls and their subfractions. Environ. Sci. Pollut. Res. 20, 5665-5672.

Dong, Y., Jinc, S., Liu, S., Xu, L., Kong, J. 2014. Effects of exogenous nitric oxide on growth of cotton seedlings under $\mathrm{NaCl}$ stress. Journal of Soil Science and Plant Nutrition. 14, 1-13.

Dong, Y.J., Wang, Z.L., Zhang, J.W., Liu, S., He, Z.L., He, M.R. 2015. Interaction effects of nitric oxide and salicylic acid in alleviating salt stress of Gossypium hirsutum L.. Journal of Soil Science and Plant Nutrition. 15, 561-573.

Durán, P., Acuña, J.J., Armada, E., López-Castillo, O.M., Cornejo, P., Mora, M.L., Azcón, R. 2016. Inoculation with selenobacteria and arbuscular mycorrhizal fungi to enhance selenium content in lettuce plants and improve tolerance against drought stress. Journal of Soil Science and Plant Nutrition. 16, 201-225.

Elstner, E.F., Heupel, A. 1976. Inhibition of nitrite formation from hydroxyl ammonium-chloride: a simple assay for superoxide dismutase. Anal. Biochem. 70, 616-620.

Ericson, M.C., Alfinito, A.E. 1984. Proteins produced during salt stress in tobacco cell cultures. Plant Physiol. 74, 506-509.

Fang, F.M., Wang, Q.C. 2000. Research progress on mercury pollution in soil. Soil Environ. 9, 326-329.

Foy, C.D., Chaney, R.L., White, M.C. 1978. The physiology of metal toxicity in plants. Annu. Rev. Plant Physiol. 29, 511-566.
Heath, R.L., Packer, L. 1968. Photoperoxidation in isolated chloroplasts: I. Kinetics and stoichiometry of fatty acid peroxidation. Arch. Biochem. Biophys. 125, 189-198.

He, H.Y., Zhan, J., He, L.F., Gu, M.H. 2012. Nitric oxide signaling in aluminum stress in plants. Protoplasma. 249, 483-492.

He, J.Y., Ren, Y.F., Chen, X.L., Chen, H. 2014. Protective roles of nitric oxide on seed germination and seedling growth of rice (Oryza sativa L.) under cadmium stress. Ecotoxicology and Environmental Safety. 108, 114-119.

Idrees, M., Naeem, M., Nasir Khan, M., Aftab, T., Khan, M.M.A., Moinuddin, 2012. Alleviation of salt stress in lemongrass by salicylic acid. Protoplasma. 249, 709-720.

Khairy, A.I.H., Oh, M.J., Lee, S.M., Kim, D.S., Roh, K.S. 2016. Nitric oxide overcomes $\mathrm{Cd}$ and $\mathrm{Cu}$ toxicity in in vitro-grown tobacco plants through increasing contents and activities of rubisco and rubisco activase. Biochimie Open. 2, 41-51.

Knudson, L.L., Tibbitts, T.W., Edwards, G.E. 1977. Measurement of ozone injury by determination of leaf chlorophyll concentration. Plant Physiol. 60, 606-608.

Kováčik, J., Klejdus, B., Babula, P., Hedbavny, J. 2015. Nitric oxide donor modulates cadmiuminduced physiological and metabolic changes in the green alga Coccomyxa subellipsoidea. Algal Research. 8, 45-52.

Liu, S.L., Yang, R.J., Pan, Y.Z., Ma, M.D., Pan, J., Zhao, Y., Cheng, Q.S., Wu, M.X., Wang, M.H., Zhang, L. 2015. Nitric oxide contributes to minerals absorption, proton pumps and hormone equilibrium under cadmium excess in Trifolium repens L. plants. Ecotoxicology and Environmental Safety. 119, 35-46.

Lou, Y.H., Luo, H.J., Hu, T., Li, H.Y., Fu, J.M. 2013.

Toxic effects, uptake, and translocation of $\mathrm{Cd}$ 
and $\mathrm{Pb}$ in perennial ryegrass. Ecotoxicology. 22, 207-214.

Manai, J., Kalai, T., Gouia, H., Corpas, F.J. 2014. Exogenous nitric oxide (NO) ameliorates salinity-induced oxidative stress in tomato (Solanum lycopersicum) plants. Journal of Soil Science and Plant Nutrition. 14, 433-446.

Neill, S.J., Desikan, R., Clarke, A., Hurst, R.D., Hancock, J.T. 2002. Hydrogen peroxide and nitric oxide as signalling molecules in plants. J Exp Bot. $53,1237-1247$.

Neill, S.J., Desikan, R., Hancock, J.T. 2003. Nitric oxide signalling in plants. New Phytology. 159, $11-35$.

Nickel, K.S., Cunningham, B.A. 1969. Improved peroxidase assay method using leuco 2,3', 6-trichloroindophenol and application to comparative measurements of peroxidatic catalysis. Anal. Biochem. 27, 292-299.

Patra, H.L., Kar, M., Mishre, D. 1978. Catalase activity in leaves and cotyledons during plant development and senescence. Biochem. Pharmacol. 172, 385-390.

Patterson, B.D., MacRae, E.A., Ferguson, I.B. 1984. Estimation of hydrogen peroxide in plant extracts using titanium (IV). Anal. Biochem. 139, 487492.

Rady, M.M., Hemida, K.A. 2015. Modulation of cadmium toxicity and enhancing cadmium-tolerance in wheat seedlings by exogenous application of polyamines. Ecotoxicology and Environmental Safety. 119, 178-185.

Saxena, I., Shekhawat, G.S. 2013. Nitric oxide (NO) in alleviation of heavy metal induced phytotoxicity and its role in protein nitration. Nitric Oxide. $32,13-20$

Stewart, R.C., Bewley, J.D. 1980. Lipid peroxidation associated with accelerated aging of soybean axes. Plant Physiol. 65, 245-248.
Tamás, L., Mistrík, I., Alemayehu, A., Zelinová, V., Bo cová, B., Huttová, J. 2015. Salicylic acid alleviates cadmium-induced stress responses through the inhibition of Cd-induced auxin-mediated reactive oxygen species production in barley root tips. Journal of Plant Physiology. 173, 1-8.

Tonamura, B. 1978. Test reactions for a stopped flow apparatus regulation of 2, 6-D and potassium ferricyanide by L-ascorbic acid. Anal. Biochem. 84, 370-383.

Wang, Q.H., Liang, X., Dong, Y.J., Xu, L.L., Zhang, X.W., Hou, J., Fan, Z.Y. 2013. Effects of exogenous nitric oxide on cadmium toxicity, element contents and antioxidative system in perennial ryegrass. Plant Growth Regul. 69, 11-20.

Wendehenne, D., Pugin, A., Klessig, D.F., Durner, J. 2001. Nitric oxide: comparative synthesis and signalling in animal and plant cells. Trends in Plant Sciences. 6, 177-183.

Xiong, J., An, L.Y., Lu, H., Zhu, C. 2009. Exogenous nitric oxide enhances cadmium tolerance of rice by increasing pectin and hemicellulose contents in root cell wall. Planta. 230, 755-765.

Xu, J., Wang, W.Y., Yin, H.X., Liu, X.J., Sun, H., Mi, Q. 2010. Exogenous nitric oxide improves antioxidative capacity and reduces auxin degradation in roots of Medicago truncatula seedlings under cadmium stress. Plant Soil. 326, 321-330.

Xu, L.L., Dong, Y.J., Kong, J., Liu, S. 2014. Effects of root and foliar applications of exogenous $\mathrm{NO}$ on alleviating cadmium toxicity in lettuce seedlings. Plant Growth Regul. 72, 39-50.

Zhang, Z.L., Di, W.J. 2003. Laboratory guide for plant physiology [M]. Higher Education Press, Beijing. 